\title{
The Computation Model and Its Test Validation of Low-Energy Plasma Collision Frequency
}

\author{
Wu Runhui ${ }^{1 *}$, Liu Jiaqi ${ }^{1}$, Liu Jutao ${ }^{1}$, Liu Xin ${ }^{1}$, Xu Hanzhong ${ }^{1}$, Liang Wei ${ }^{1}$ \\ ${ }^{1}$ National Key Laboratory of Science and Technology on Test Physics \& Numerical Mathematical, Beijing \\ P.0.9200-76-7, Beijing 100076, China.
}

* Corresponding author. Tel.: 13522078693; email: runhui_wu@aliyun.com, rhonda20120322@sohu.com Manuscript submitted August 10, 2014; accepted January 15, 2015.

doi: 10.17706/ijapm.2015.5.2.94-104

\begin{abstract}
In this paper, we propose the computational model of plasma collision frequency based on the plasma particle component. The computational model of collision between electrons and neutral particles is validated through the parallel panel collision frequency experiment in which the electrons are simulated by the laser. The electromagnetic wave propagation experiment in plasma under the high temperature shock wave tube environment proves the effectiveness of plasma collision frequency model. Results from comparison between simulation and experiment indicate that the proposed method is able to provide comprehensive description of multi-component plasma collision phenomenon and high accuracy in the collision frequency computation among electrons, ions and neutral particles. The research achievement is helpful applied to evaluate the effect of plasma collision absorption.
\end{abstract}

Key words: Collision frequency, low-energy plasma, validation test.

\section{Introduction}

Many efforts are put on the research about electromagnetic energy absorption of plasmas [1]-[7]. Collision absorption is one of the representative mechanism in plasma electromagnetic absorption. The plasma collision frequency is the critical parameter that describes the performance of energy absorption. Collision frequency is determined by various factors including the plasma generation and component combination. The accuracy of collision frequency evaluation has great influence on the electromagnetic energy absorption performance evaluation. Therefore, the model of plasma collision frequency and the corresponding validation experimental scheme is fundamental to plasma collision absorption research.

In this paper, we propose the computational model of collision frequency corresponding to electrons versus ions, electrons versus neutral particles and the total collision frequency. The model is validated through the parallel panel experiment of electron and neutral particle collision frequency stimulated by the laser. The total collision frequency model is proved to be effective through the electromagnetic wave propagation experiment in plasma under high temperature shock wave tube environment. Results compared indicate that the proposed model is suitable for the collision frequency computation in plasma collision absorption research.

\section{Computational Model of Low Energy Plasma Collision Frequency}

Plasma is a kind of quasi-neutral gas which is composed of electrons, ions and neutral particles [8]. 
Plasma component determines the component of ions and neutral particles. As for the computational model, plasma's collision frequency contains the collision of electrons versus ions, electrons versus electrons, electrons versus neutral particles, ions versus ions and ions versus neutral particles [9]. Collisions of electrons versus electrons, ions versus ions satisfy the law of momentum conservation, the collision absorption of electromagnetic wave is not influenced by plasma's collision model. Due to the fact that ion's mass is much larger than that of electron, the collision frequency of ion versus electron is usually three orders of magnitude less than that of ions versus neutral particles, the former one is usually neglected. The main contribution of electromagnetic wave collision absorption comes from the collision of electrons versus ions, electrons versus neutral particles, which are also the major concern in the computational model.

\subsection{Collision between Electrons and Ions}

Coulomb model is usually applied in the calculation of electrons versus ions collision frequency. The mathematical formula is [4], [8]:

$$
v_{\mathrm{ei}}=\frac{4 \sqrt{2 \pi}}{3} N_{i}\left(\frac{Z e^{2}}{k T_{e}}\right)^{2}\left(\frac{k T_{e}}{m_{e}}\right)^{\frac{1}{2}} \ln \wedge
$$

where $N_{i}$ and $Z$ are the number density of ions and charge number; $m_{e}, e$ and $T_{e}$ are the mass, electric charge and the temperature of the electron; $\ln \Lambda$ is the coulomb logarithm, given by:

$$
\ln \Lambda=\ln \frac{4 \kappa T_{e}}{\gamma^{2} Z e^{2} k_{e}}-\frac{k_{e}^{2}+k_{i}^{2}}{k_{i}^{2}} \ln \frac{\sqrt{k_{e}^{2}+k_{i}^{2}}}{k_{e}}
$$

where $k_{i}^{2}$ and $k_{e}^{2}$ denote the nexus between electron energy and number density, $k_{i}^{2}$ and $k_{e}^{2}$ equation can be obtained by:

$$
\begin{aligned}
& k_{i}^{2}=\frac{4 \pi \mathrm{N}_{i} Z^{2} e^{2}}{\kappa T_{i}} \\
& k_{e}^{2}=\frac{4 \pi \mathrm{N}_{e} e^{2}}{\kappa T_{e}}
\end{aligned}
$$

where $K T_{i}$ and $v K T_{e}$ are the temperature of ion and electron, $N_{i}$ and $N_{e}$ are the ion and electron density.

According to the above formulas, the collision frequency of electron versus ion could be calculated with the density and temperature of electron and ion respectively.

\subsection{Collision of Electrons versus Neutral Particles}

The collision frequency of electrons versus neutral particles is related to the category of neutral particle's component, and the collision cross section under various electron temperatures. The collision frequency ven could be formulated as [8]:

$$
v_{\mathrm{en}}=<\sigma v>N_{n}
$$

where $\sigma$ is the collision cross section of electrons versus neutral particles collision, $v$ is the electron moving velocity, $N_{n}$ is the neutral particle number density, $\langle\sigma v\rangle$ is the effective velocity collision 
coefficient, whose calculation formula is [8]:

$$
<\sigma v>=\int_{0}^{\infty} \sigma v f(v) \mathrm{d} v
$$

where $v$ is the electron moving velocity, $f(v)$ is the distribution function of electron velocity. The electron velocity usually follows the Maxwell distribution for thermal equilibrium plasma. For the isotropic plasma, one dimensional Maxwell distribution is usually taken. $f(v)$ is calculated as [8]:

$$
f(v)=A \exp \left(\frac{1}{2} m_{\mathrm{e}} v^{2} / k T_{e}\right)
$$

where $A$ is the constant, $k$ is the Boltzmann constant, and it is equal to $1.38 \times 10^{-23} \mathrm{~J} / \mathrm{K}$, and $T_{e}$ is the electron temperature, $A$ is calculated by:

$$
A=n_{e}\left(\frac{m_{e}}{2 \pi k T_{e}}\right)^{\frac{1}{2}}
$$

If there are many kinds of neutral particle components, suppose the kind number is $n$, and the collision frequency of electrons and neutral particles is calculated by:

$$
v_{\mathrm{en}}{ }^{t}=\sum_{i=1}^{n}<\sigma_{i} v_{i}>N_{i}
$$

\subsection{Total Collision Frequency of Plasma}

For elastic collision, the total collision frequency of electrons, ions and $\mathrm{n}$ types of neutral particles is the linear combination of coulomb collision by electrons versus ions and the elastic collision of electrons versus neutral particles, which is calculated as:

$$
v_{t}=v_{e i}+v_{e n}^{t}
$$

Based on the model of total collision frequency, it is clear that the total collision frequency is available with known plasma component type, the temperature and density of electrons and ions, in addition to the component neutral particle density.

\section{Validation Experiment of Collision Frequency Computation Model}

The collision frequency computation model in part 2 is widely suitable for plasma with various types of neutral particles, charged electrons and other particles. The accuracy of collision frequency calculation has great influence on the reliability of plasma's electromagnetic wave collision absorption effect analysis. Therefore, it is necessary to validate the proposed model through various ground experiments. In this paper, the calculation model of collision frequency is validated by two kinds of experiments:

1) The first one is the parallel panel experiment which uses the collision of laser stimulated electrons and neutral particles to validate the calculation model of collision between electrons and various neutral particles.

2) The second one is the high temperature shock wave tube experiment which validates the plasma total 
collision frequency calculation model.

\subsection{Laser Stimulated Electron and Neutral Particle Collision Parallel Panel Experiment}

\subsubsection{Experiment device and test program [9]}

The experiment consists of laser source, valve system, sample pool system, signal detection and process system, which is demonstrated in Fig. 1.

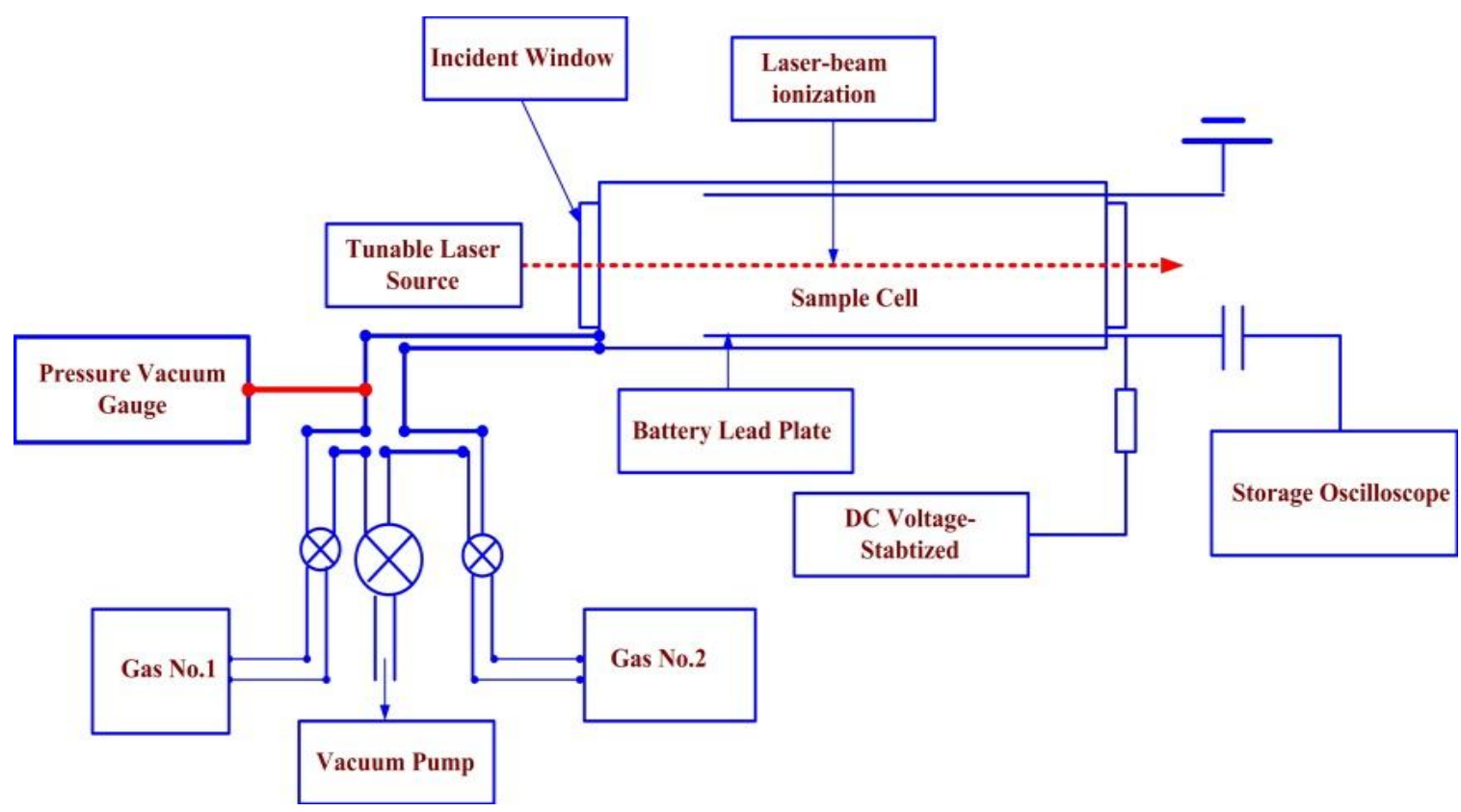

Fig. 1. The basic composition of experiment device.

As in Fig.1, there are two parallel electrode panels with specified voltage(10 1000V) in the sample pool filled with the gas that will be testified. The impulse laser incidence window is on the flank of the sample tool. The neutral gas is injected through the valve system. The generated laser pulse ionizes the neutral gas in the sample pool so that the ionized electrons shifted from the middle of the panels to the positive panel under the applied electric field. Electrons shifting process results in the collision with neutral particles. The electron shifting velocity could be calculated by measuring the time which electrons arrive at the positive panel. The collision frequency between electrons and various component neutral particles is calculated through the temperature and pressure of the sample pool. The data processing detail is as follows:

1) The electron's macroscopic shifting velocity $v_{d}$ is calculated by directly measuring the electron's arrival time tm on the positive panel:

$$
v_{d}=\frac{d_{e}}{2 t_{m}}
$$

where $d_{e}$ is the spacing of the electrode panels.

2) Calculate the elastic collision cross section $\sigma_{0}$ of electrons and molecules using the neutral gas component diameter $d$ :

$$
\sigma_{0}=\pi d^{2}
$$

3) Calculate the neutral gas density $N_{n}$ using the measured pressure and temperature of the gas: 


$$
N_{n}=\frac{P}{k_{B} T}
$$

where $P$ is the pressure of the sample tool, $k_{B}$ is the Boltzmann constant, $T$ is the temperature of the sample pool.

4) In the condition of elastic collision, calculate the electron thermal motion velocity $v_{t h}$ using electron shifting velocity $v_{d}$, elastic collision cross section $\sigma_{0}$ and neutral particle density $N_{n}$ :

$$
v_{t h}=\frac{e}{m_{e} N_{n} \sigma_{0} v_{d}} E
$$

5) Calculate the electron and neutral particle collision frequency $v_{e n}$ using electron thermal motion velocity $v_{t h}$, elastic collision cross section $\sigma_{0}$ and neutral particle density $N_{n}$ :

$$
v_{e n}=N_{n}<\sigma_{0} v_{t h}>
$$

\subsubsection{Comparison between experiment and simulation}

The collision frequency experiments of electrons versus argon gas, nitrogen gas and potassium atomics is accomplished utilizing the device and model in 3.1.1. The collision frequency computation of electrons

\begin{tabular}{|c|c|c|c|c|c|}
\hline Parameter name & No.1 & No.2 & No.3 & No.4 & No.5 \\
\hline Auxiliary gap(mm) & 10 & 10 & 10 & 10 & 10 \\
\hline Electrode voltage(V) & 89.2 & 89.2 & 89.2 & 89.2 & 89.2 \\
\hline Sample cell pressure(Pa) & 10000 & 20000 & 30000 & 40000 & 50000 \\
\hline \multicolumn{6}{|l|}{ Time of the electrons reach the positive } \\
\hline electrode plate(ns) & 249 & 267 & 332 & 390 & 450 \\
\hline Electron thermal velocity $(\mathrm{cm} / \mathrm{s})$ & $3.48 \times 10^{4}$ & $3.48 \times 10^{4}$ & $3.48 \times 10^{4}$ & $3.48 \times 10^{4}$ & $3.48 \times 10^{4}$ \\
\hline Electron temperature(K) & 16540 & 15171 & 14140 & 13414 & 12804 \\
\hline Argon particle number density $\left(\mathrm{cm}^{-3}\right)$ & $0.25 \times 10^{19}$ & $0.49 \times 10^{19}$ & $0.74 \times 10^{19}$ & $0.99 \times 10^{19}$ & $1.24 \times 10^{19}$ \\
\hline Collision cross section $\left(\mathrm{cm}^{2}\right)$ & $3.02 \times 10^{-16}$ & $3.02 \times 10^{-16}$ & $3.02 \times 10^{-16}$ & $3.02 \times 10^{-16}$ & $3.02 \times 10^{-16}$ \\
\hline \multicolumn{6}{|l|}{ Collision frequency of experimental } \\
\hline measurement $v_{\exp }(\mathrm{Hz})$ & $2.9 \times 10^{7}$ & $5.15 \times 10^{7}$ & $7.8 \times 10^{7}$ & $1 \times 10^{8}$ & $1.3 \times 10^{8}$ \\
\hline $\begin{array}{l}\text { Collision frequency of simulation } v_{\text {the }} \\
\qquad(\mathrm{Hz})\end{array}$ & $2 \times 10^{7}$ & $3 \times 10^{7}$ & $4.1 \times 10^{7}$ & $5.26 \times 10^{7}$ & $6.5 \times 10^{7}$ \\
\hline Simulation error $v_{\text {exp }} / v_{\text {the }}$ & 1.45 & 1.7 & 1.9 & 1.9 & 2 \\
\hline
\end{tabular}
versus neutral particles is also achieved with results comparison illustrated from Table 1 to Table 3.

Table 1. The Experiment and Simulation Results of Collision Frequency (Electrons Versus Argon) 
Table 2. The Experiment and Simulation Results of Collision Frequency (Electrons Versus Nitrogen)

\begin{tabular}{|c|c|c|c|c|c|}
\hline Parameter name & No.1 & No.2 & No.3 & No.4 & No.5 \\
\hline Auxiliary gap(mm) & 10 & 10 & 10 & 10 & 10 \\
\hline Electrode voltage(V) & 89.2 & 89.2 & 89.2 & 89.2 & 89.2 \\
\hline Sample cell pressure(Pa) & 10000 & 20000 & 30000 & 40200 & 50000 \\
\hline $\begin{array}{l}\text { Time of the electrons reach the positive } \\
\text { electrode plate(ns) }\end{array}$ & 535 & 665 & 738 & 851 & 940 \\
\hline Electron thermal velocity $(\mathrm{cm} / \mathrm{s})$ & $4.16 \times 10^{4}$ & $4.16 \times 10^{4}$ & $4.16 \times 10^{4}$ & $4.16 \times 10^{4}$ & $4.16 \times 10^{4}$ \\
\hline Electron temperature(K) & 3282 & 2941 & 2779 & 2653 & 2567 \\
\hline Argon particle number density $\left(\mathrm{cm}^{-3}\right)$ & $0.29 \times 10^{19}$ & $0.55 \times 10^{19}$ & $0.81 \times 10^{19}$ & $1.06 \times 10^{19}$ & $1.3 \times 10^{19}$ \\
\hline Collision cross section $\left(\mathrm{cm}^{2}\right)$ & $3.76 \times 10^{-16}$ & $3.76 \times 10^{-16}$ & $3.76 \times 10^{-16}$ & $3.76 \times 10^{-16}$ & $3.76 \times 10^{-16}$ \\
\hline $\begin{array}{l}\text { Collision frequency of experimental } \\
\text { measurement } v_{\exp }(\mathrm{Hz})\end{array}$ & $4.5 \times 10^{7}$ & $8.6 \times 10^{7}$ & $1.3 \times 10^{8}$ & $1.7 \times 10^{8}$ & $2 \times 10^{8}$ \\
\hline $\begin{array}{l}\text { Collision frequency of simulation } v_{\text {the }} \\
\qquad(\mathrm{Hz})\end{array}$ & $1 \times 10^{8}$ & $1.9 \times 10^{8}$ & $2.9 \times 10^{8}$ & $3.7 \times 10^{8}$ & $4.3 \times 10^{8}$ \\
\hline Simulation error $v_{\exp } / v_{\text {the }}$ & 0.45 & 0.453 & 0.448 & 0.46 & 0.465 \\
\hline
\end{tabular}

Table 3. The Experiment and Simulation Results of Collision Frequency (Electrons Versus Potassium)

\begin{tabular}{|c|c|c|c|}
\hline Parameter name & No.1 & No.2 & No.3 \\
\hline Auxiliary gap (mm) & 10 & 10 & 10 \\
\hline Electrode voltage(V) & 88.8 & 88.8 & 88.8 \\
\hline Sample cell pressure $(\mathrm{Pa})$ & 266 & 1064 & 2394 \\
\hline $\begin{array}{l}\text { Time of the electrons reach the positive electrode } \\
\qquad \text { plate(ns) }\end{array}$ & 100 & 190 & 229 \\
\hline Electron thermal velocity $(\mathrm{cm} / \mathrm{s})$ & $5.56 \times 10^{4}$ & $6.02 \times 10^{4}$ & $6.45 \times 10^{4}$ \\
\hline Electron temperature $(\mathrm{K})$ & 7362 & 3558 & 2414 \\
\hline Potassium atom number density $\left(\mathrm{cm}^{-3}\right)$ & $0.34 \times 10^{17}$ & $0.115 \times 10^{18}$ & $0.224 \times 10^{18}$ \\
\hline Collision cross section $\left(\mathrm{cm}^{2}\right)$ & $16.2 \times 10^{-16}$ & $16.2 \times 10^{-16}$ & $16.2 \times 10^{-16}$ \\
\hline $\begin{array}{l}\text { Collision frequency of experimental measurement } v_{\exp } \\
\qquad(\mathrm{Hz})\end{array}$ & $3 \times 10^{6}$ & $1.12 \times 10^{7}$ & $2.34 \times 10^{7}$ \\
\hline Collision frequency of simulation $v_{\text {the }}(\mathrm{Hz})$ & $5.4 \times 10^{6}$ & $2.5 \times 10^{7}$ & $5.63 \times 10^{7}$ \\
\hline Simulation error $v_{\text {exp }} / v_{\text {the }}$ & 0.56 & 0.448 & 0.416 \\
\hline
\end{tabular}

The results comparison indicates the good match between measurement and simulation in electron versus neutral particle collision frequency. The maximum difference is within three times. This proves the collision frequency model of electrons versus neutral particles.

\subsection{Plasma Total Collision Frequency Experiment with High Temperature Shock Wave Tube}




\subsubsection{Experiment device and test program}

The experiment device consists of high voltage part, low voltage part, diaphragm, vacuum system, plasma sheath transmission test part, corresponding test systems as demonstrated in Fig. 2 [10], [11]. The plasma sheath will be generated in the test part of Fig. 2 when the high temperature shock wave tube is activated. The signal energy attenuation and phase change information of electromagnetic wave in plasma sheath could be collected by vector network analyzer. The attenuation coefficient and electron density in electromagnetic wave propagation path could be calculated through energy attenuation and phase difference. The total plasma collision frequency is the function of attenuation coefficient alpha and electron density ne.

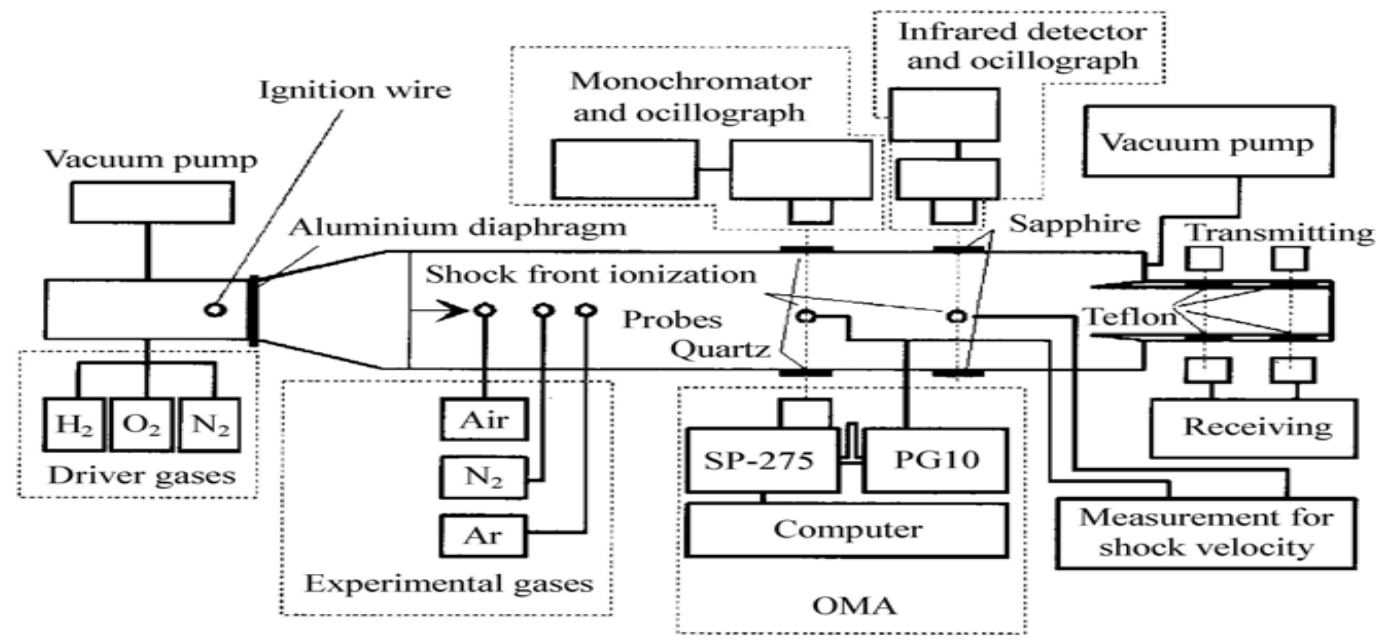

Fig. 2. Schematic diagram of $\Phi 800 \mathrm{~mm}$ shock tube and instrumentation arrangement.

This program is theoretically based on the fact that when the plane electromagnetic wave propagates in the infinite uniform plasma without magnetic field, the power change of electromagnetic wave is described as:

$$
P=P_{0} \exp (j \omega t-\gamma z)
$$

where $P_{0}$ is the power of incidence wave, $\omega$ is the angular frequency, $\gamma$ is the propagation constant, which could be demonstrated by attenuation coefficient and phase constant $\beta$ :

$$
\gamma=\alpha+j \beta
$$

where $\alpha$ is available through vector network analyzer, it is the function of angular frequency $\omega$, plasma characteristic angular frequency $\omega_{p}$ and plasma collision frequency $\mathrm{v}$, and $\alpha$ is given by:

$$
\alpha=\frac{\omega}{c}\left\{\frac{1}{2}\left[\left(1-\frac{\omega_{p}^{2}}{\omega^{2}+v^{2}}\right)^{2}+\left(\frac{\omega_{p}^{2}}{\omega^{2}+v^{2}} \cdot \frac{v}{\omega}\right)^{2}\right]^{\frac{1}{2}}-\frac{1}{2}\left(1-\frac{\omega_{p}^{2}}{\omega^{2}+v^{2}}\right)\right\}^{\frac{1}{2}}
$$

where $c$ is the velocity of light propagation, the relationship between plasma characteristic angular 
frequency $\omega_{p}$ and plasma characteristic frequency $f_{p}$ is obtained as:

$$
\omega_{p}=2 \pi f_{p}
$$

The relationship between plasma characteristic frequency $f_{p}$ and electron density $n_{e}$ is given by:

$$
f_{p}=9000 \sqrt{n_{e}}
$$

where the units of $f_{p}$ and $n_{e}$ are $\mathrm{Hz}$ and $\mathrm{cm}^{-3}$ respectively.

When the electromagnetic wave propagates through plasma with thickness of $z$ the refraction phase change $\Delta \phi_{p}$ is expressed as [11]:

$$
\Delta \phi_{p}=\int_{0}^{z} \frac{2 \pi(n-1)}{\lambda} d x
$$

where $\lambda$ is the wave length, in the infinite uniform plasma without magnetic field, refraction rate $n$ is:

$$
n=\left\{\frac{1}{2}\left(1-\frac{\omega_{p}^{2}}{\omega^{2}+v^{2}}\right)+\frac{1}{2}\left[\left(1-\frac{\omega_{p}^{2}}{\omega^{2}+v^{2}}\right)^{2}+\left(\frac{\omega_{p}^{2}}{\omega^{2}+v^{2}} \frac{v}{\omega}\right)^{2}\right]^{\frac{1}{2}}\right\}^{\frac{1}{2}}
$$

Use Eq.(21) in Eq.(20), $\Delta \phi_{p}$ can be obtained as:

$$
\Delta \phi_{p}=\frac{\pi z n_{e}}{\lambda n_{e c}}
$$

where $n_{e c}$ is the total reflection cut-off electron density on the plasma boundary, and it should be expressed as:

$$
n_{e c}=1.23 \times 10^{10} f^{2}
$$

where $f$ is the electromagnetic wave frequency. The average value of plasma electron density $n_{e}$ could be calculated by Eq.(22) and Eq.(23):

$$
n_{e}=1.184 \times 10^{2} \times \frac{f \Delta \varphi_{p}}{z}
$$

Therefore, the total plasma collision frequency on electromagnetic wave propagation path is available with parameters $\alpha$ and $\Delta \varphi_{p}$ measured from the experiment.

\subsubsection{Comparison between experiment and simulation}


The result comparison between experiment and simulation under three typical conditions are illustrated in Table 4.

Table 4. The Result Comparison between Experiment and Simulation under Three Typical Condition

\begin{tabular}{|c|c|c|c|}
\hline Parameter name & No.1 & No.2 & No.3 \\
\hline One-zone pressure $\mathrm{P}_{1}(\mathrm{~Pa})$ & 13.3 & 133 & 133 \\
\hline The frequency of the incident electromagnetic wave $(\mathrm{GHz})$ & 37.5 & 10 & 37.5 \\
\hline Shock Mach & 12.3 & 9.5 & 10.5 \\
\hline Two-zone temperature $\mathrm{T}_{2}(\mathrm{~K})$ & 4200 & 3250 & 3510 \\
\hline Plasma electrons density $\left(\mathrm{cm}^{-3}\right)$ & $5 \times 10^{12}$ & $5 \times 10^{10}$ & $2 \times 10^{11}$ \\
\hline $\begin{array}{l}\text { Total collision frequency of experimental measurement } v_{\text {exp }} \\
\qquad((\mathrm{Hz})\end{array}$ & $2 \times 10^{10}$ & $1 \times 10^{10}$ & $2 \times 10^{10}$ \\
\hline Total collision frequency of simulation $v_{\text {the }}(\mathrm{Hz})$ & $2.39 \times 10^{10}$ & $1.65 \times 10^{10}$ & $1.9 \times 10^{10}$ \\
\hline Simulation error $v_{\text {exp }} / v_{\text {the }}$ & 0.84 & 0.61 & 1.05 \\
\hline
\end{tabular}

The contents in Table 4 indicate the good match between experiment and simulation, which validates the proposed total collision frequency models.

\section{Conclusion}

The plasma collision frequency model validation through two experiments indicates that:

1) The collision frequency model of electrons and neutral particles based on the effective collision coefficient is proved to be effective.

2) According to the complexity of electrons, ions and neutral particles composition, the plasma total collision frequency is approximated as the linear combination of collision frequency from electrons versus ions, electrons versus neutral particles. Experiment result validates the proposed model.

In conclusion, the collision frequency model proposed in this paper could give accurate result under various composition conditions. Its application in plasma's electromagnetic wave collision absorption simulation has great significance in plasma collision absorption research.

\section{Acknowledgments}

The authors gratefully acknowledge the support from the National Natural Science Foundation of China under Grant No. 61302029, The project name is "The Research of the Insufflation Plasma Diffusing Law and its Stealth Models"

\section{References}

[1] Wu, C. G., Wu, W. Y., \& Gong, Y. C. (2011). The collision absorption of electromagnetic wave by cold plasma. Journal of Hebei University of Science and Technology, 32, 201-203.

[2] Santoru, Joseph, Gregoire, \& Daniel, J. (2010). Electromagnetic-wave absorption in highly collisional plasmas. Journal of Applied Physics, 74(6), 3736-3743.

[3] Gregolre, D. J., Santoru, J., \& Schumacher, R. W. (1992). Electromagnetic-wave propagation in unmagnetized plasmas. $A D-A 250,710,7-10$.

[4] Wu, R. H., Ren, A. M., \& Gang, M. (2008). Research on the plasma collisional absorption of electromagnetic-wave upper the aerosphere. Journal of CAEIT, 3, 535-536. 
[5] Xie, S. G., \& Lin, D. L. (2008). Broadband Absorption of Electromagnetic-waves in Collisional Plasma. Journal of Telemetry, Tracking and Command, 29, 4-8.

[6] Song, F. L., Cao, J. X., \& Wang, G. (2005). A model of the absorption of electromagnetic-waves by weakly ionized plasma and the numerical calculation. ACTA Physica Sinica, 54, 807-810.

[7] Zheng, Y. G. (2008). The Study of Electromagnetic Scattering on Plasma. Master degree, Xi'anUniversity of Electron and Technology.

[8] The physics theories of plasma. (1981). Demotic Education Publishing House, China.

[9] Zhang, L. M., Chen, H. Z., et al. (2001). Study on photochemical Reaction channels of acetone in the range 243 263nm. Chinese Journal of Chemical Physics, 14(2), 135-136.

[10] Zhu, N. Y., Li, X. F., Huang, L. S., Yu, X. L., \& Yang, Q. S. (2004). An investgation of electromagnetic wave propagation in plasma by shock tube. ACTA Mechanica Sinica, 20(3), 212-218.

[11] (1965). Plasma Diagnostics with Microwaves. New York: Jehn Wiley and Sons.

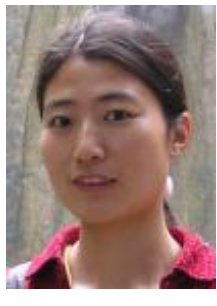

Wu Runhui was born in Yujiang, Jiangxi province, P.R.Chnia, on March 9, 1974. She received the B. S. degree from the Hefei Institute of Technology, Hefei, China, and the M. S. degree from University of Science and Technology of China. Her research interests include the plasma generator, such as DC discharge and RF discharge; the theory about the electromagnetic-wave propagation in the plasma; the study of the physical model for the collision mechanism in different discharge plasma; the application of plasma, such as plasma antenna.

Her study interests focused on plasma generator, plasma diffusion in opening space, plasma EM scattering, the test technology about the collisional absorption of electromagnetic-wave in plasma etc.

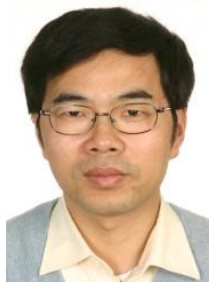

Liu Jiaqi was born in Yueyang, Hunan province, P.R.Chnia, on Oct. 20, 1963. He received the B. S. degree, M. S. degree from National University of Defense Technology. In 2006, he earned the Ph.D. degree in from Beijing University of Aeronautics \& Astronautics, Beijing, China.

His study interests focused on EM scattering, computational electromagnetics, reaction of plasma and EM wave, periodic structure, etc.

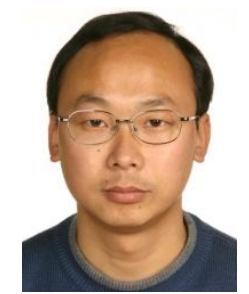

Liu Jutao was born in Xixiang, Shanxi province, P.R.Chnia, on Sep. 7, 1976. He received M. S. degree from China University of Petroleum, Beijing, China.

His major field of study focuses on response of materials with electromagnetic (EM) wave, Frequency selective surfaces, active periodic structures, EM scattering, plasma generation, diagnosis, and its reaction with EM wave.

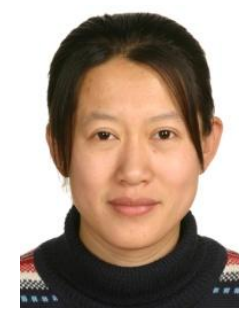

Liu Xin was born in Fushun, Liaoning province, P.R.Chnia, on July. 12, 1975. She received the B. S. and M.S degree from the Haerbin Institute of Technology, Haerbini, China,. and the M.S. degree from Her research interests include the plasma generator, such as DC discharge and RF discharge; the theory about the electromagnetic-wave propagation in the plasma; the study of the physical model for the collision mechanism in different discharge plasma; the application of plasma, such as plasma antenna.

Her study interests focused on plasma generator, plasma diffusion in opening space, plasma EM scattering, the test technology about the collisional absorption of electromagnetic-wave in plasmaetc. 


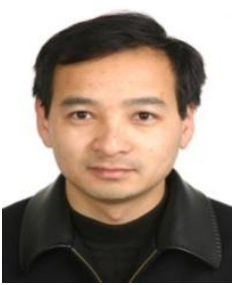

Xu Hanzhong was born in Yanchen, Jiangsu province, P.R.Chnia, on Aug. 16, 1975. He received the B. S. degree, M. S. degree from Nanjing University of Science \& Technology. His major field of study focuses on response of materials with electromagnetic (EM) wave, Frequency selective surfaces, active periodic structures, EM scattering, plasma generation, diagnosis, and its reaction with EM wave.

His study interests focused on EM scattering, Computational electromagnetics, reaction of plasma and EM wave, periodic structure, etc.

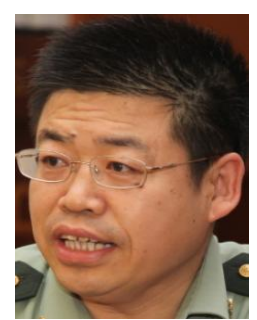

Liang Wei was born in Changsha, Hunan province, P.R.Chnia, on Dec. 20, 1966. He received the B. S. degree, M. S. degree and Ph.D. degree from Beijing University. His major field of study focuses on response of materials with electromagnetic (EM) wave, frequency selective surfaces, active periodic structures, EM scattering, plasma generation. His major field of study focuses on response of materials with electromagnetic (EM) wave, Frequency selective surfaces, active periodic structures, EM scattering, plasma generation, diagnosis, and its reaction with EM wave. 